\section{The Stability of Pt Nanofilaments in a Au-matrix Composite}

\author{
K Wongpreedee ${ }^{1}$ and AM Russell ${ }^{2}$ \\ ${ }^{1}$ Materials Science (Gems and Jewelry) program, \\ Department of General Science, Faculty of Science, \\ Srinakharinwirot University, Bangkok, Thailand, 10110 \\ E-mail: kageeporn@swu.ac.th \\ ${ }^{2}$ Department of Materials Science \& Engineering and \\ Ames Laboratory of the U.S. Dept. of Energy, lowa \\ State University, Ames, IA, USA, 50011
}

\begin{abstract}
Au-7 at.\% Pt Deformation-processed Metal Metal Composite (DMMC) was isothermally annealed at various times and temperatures. Avrami and Arrhenius relations were used to evaluate the kinetics of the transformation from the initial metastable nanofilamentary microstructure to the equilibrium homogeneous solid solution condition. Results indicated that the activation energy for this transformation was $167 \pm 6 \mathrm{~kJ} / \mathrm{mol}$. This activation energy is lower than activation energies reported in the literature for planar interface, single crystal Au-Pt diffusion couple experiments. The effects of chemical potential, surface curvature, and strain effects on the empirical activation energies determined in the DMMC of this study are discussed. These factors are thought to cause the DMMC systems to reach equilibrium faster.
\end{abstract}

\section{Keywords}

Gold, composite, transformation, capillary, activation energy

\section{Introduction}

During the past quarter century, several Deformationprocessed Metal Metal Composites (DMMC's) have been developed in Cu-Nb [1], Cu-Ag [2], Au-Ag [3], and other binary systems [4]. In contrast to conventional metal matrix composites reinforced by ceramic filaments, DMMC's are produced by cold working specimens comprised of two metal phases to reductions in area greater than 99.99\%. DMMC's have an advantage for producing high-strength electrical wires since the metal reinforcement filaments, unlike ceramic reinforcements, contribute substantially to conductivity.

DMMC's can be produced from co-melted metals that are mutually immiscible in the solid phase without intermetallic compound formation, or they can be produced from powder metallurgy compacts. In either case it is necessary that the two metals are ductile and possess approximately equal flow stresses. The typical microstructure of axisymmetrically deformed DMMC's is cylindrical filaments for fcc filamentfcc matrix systems or ribbon-shaped filaments for bcc filament-fcc matrix systems as shown in Fig. 1. The shape of the filaments in DMMC's results from texture development during deformation that causes plane strain deformation of bcc filaments but permits triaxial strain in fcc filaments [4].

Although considerable attention has been devoted to explaining the high strength of DMMC's [1-4], less attention has been devoted to study the changes in DMMC microstructure and properties that result from prolonged exposure to elevated temperatures during service. Since the DMMC microstructure is metastable, it is vulnerable to filament coarsening due to energy minimization from the large driving force of the capillary effect.

Thermal instability has been studied in DMMC's by measuring changes in mechanical properties and filament shape. Spitzig, et alia [5], studied tensile properties after high temperature annealing of Cu-Ta and Cu-Nb DMMC's. They found that Ta second phase filaments display slower coarsening than $\mathrm{Nb}$ filaments, presumably due to slower boundary diffusion in Ta. They also found that both DMMC's showed substantial loss of room temperature strength after anneals at $873 \mathrm{~K}$. More recently, Lee, et alia [6], performed tensile tests and examined the fracture behavior on a Cu$\mathrm{Cr}$ DMMC in the temperature range 203 to $873 \mathrm{~K}$ to study the load transfer between the $\mathrm{Cr}$ filaments and the Cu matrix. They suggested that the $\mathrm{Cu}$ becomes much more plastic (softer) than the $\mathrm{Cr}$ with increasing temperature by observing microstructure damage near the filament ends. One of the first studies of the shape instability of DMMC's was performed by Malzahn Kampe, et alia [7], on Cu-Fe and NiW DMMC's. In that study coarsening was observed to be a sequential process beginning with a primary shape instability condition, followed by cylinderization, boundary splitting, and edge spheroidization. The microstructural change ended when the second phase was completely converted from a filamentary or lamellar morphology to spheres dispersed in 


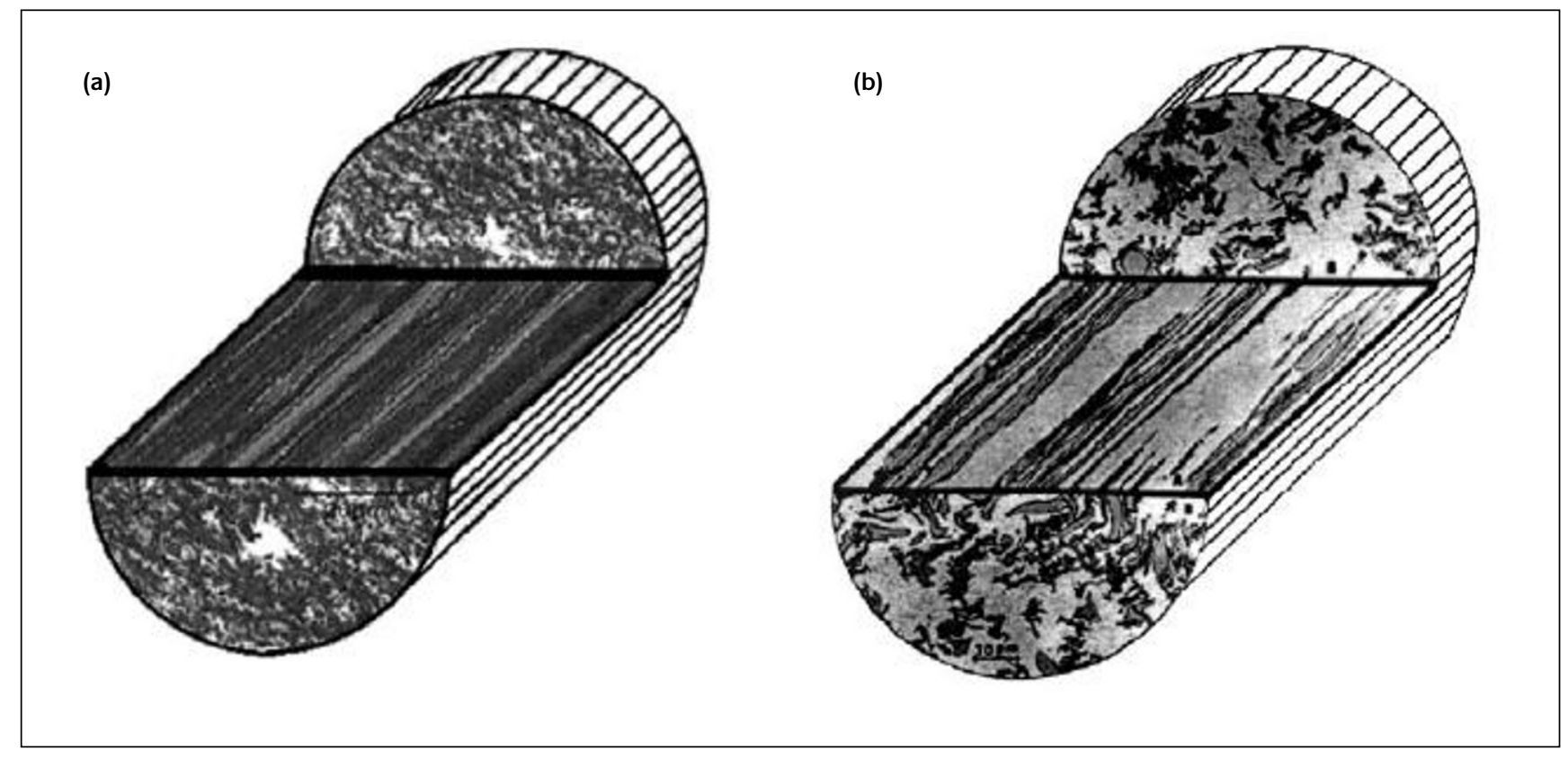

Figure 1

A depiction of typical microstructures in axisymmetrically deformed MMC's. a) In fCc matrix - fcc reinforcing phase composites, the reinforcing phase particles change during deformation processing from equiaxed powder particles to filaments that are approximately cylindrical in shape [4]. b) In fcc matrix - bcc reinforcing phase composites, ribbon-shaped filaments result from the plane straining mode present in the heavily textured bcc phase [4]

the matrix.

In a more recent study, Xu, et alia [8], observed spheroidization of $\mathrm{Sn}$ filaments in an Al-Sn DMMC, a process that occurred even at room temperature due to the low

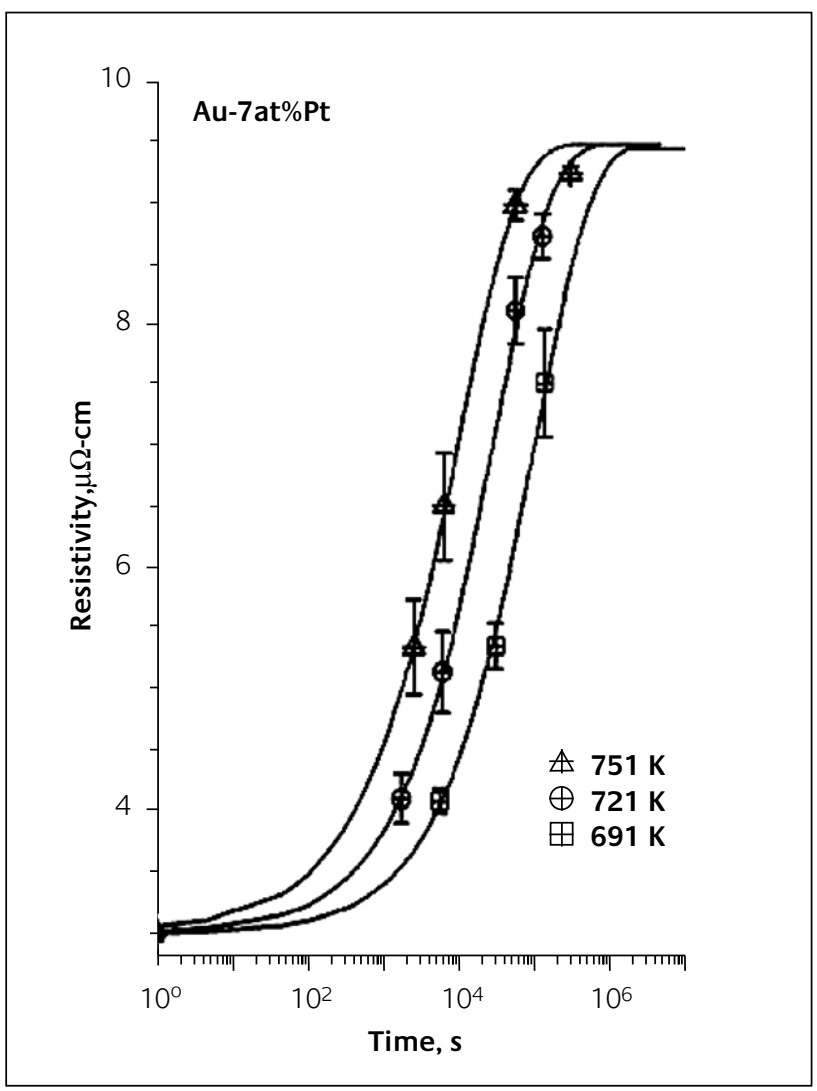

\section{Figure 2}

Plot of electrical resistivity versus annealing time for Au-7at.\%Pt DMMC annealed at the three temperatures marked. Error bars indicate \pm one standard deviation melting point of Sn. Xu observed that filamentary coarsening began when the second phase had a sinusoidal shape perturbation along its length that reached a critical wavelength value. Once that occurred, the instability progressed to spheroidization due to a chemical potential difference from the Gibbs-Thompson driving force. His assumption was correlated with earlier theoretical calculations of instability of cylindrically curved surface perturbations from Rayleigh [9] and of chemical potential as a driving force of grain boundary grooving from Mullins [10].

In this study the rate of transformation of a Au-Pt DMMC was measured and analyzed. The Au-Pt DMMC was developed for potential use for interconnect wires of microprocessors and for wear-resistant switch contacts. Johnson-Mehl-Avrami (JMA) and Arrhenius equations were used to analyze the activation energy of filament transformation. The Au-Pt system differs from the systems examined in previous coarsening studies because Pt is completely soluble in the Au matrix in the solid state. At elevated temperatures the Pt filaments do not change shape; they simply dissolve into the matrix. Thus, the Au-Pt DMMC transforms from a metastable filamentary structure to the equilibrium homogeneous solid solution following the JMA equation for this physical change. This means that there is no effect of energy due to shape conversion to spheres because spheres do not form. It also means that no critical wavelength or other perturbation criteria must be satisfied before change begins in the filaments; all Pt filament shapes are inherently unstable in a Au matrix. However, the capillary effect of the second phase will be expected to affect the rate of transformation as a driving force mechanism. It is hoped that the results of this study will improve understanding of the nature of instability rate transformations in DMMC's. 


\section{Experimental procedures}

The Au-Pt binary system is well-suited to DMMC production because it contains no intermetallic compounds, both metals are highly ductile, and the flow stress of Pt is fairly similar to that of Au. A Au-7 at.\%Pt powder blend was mixed and

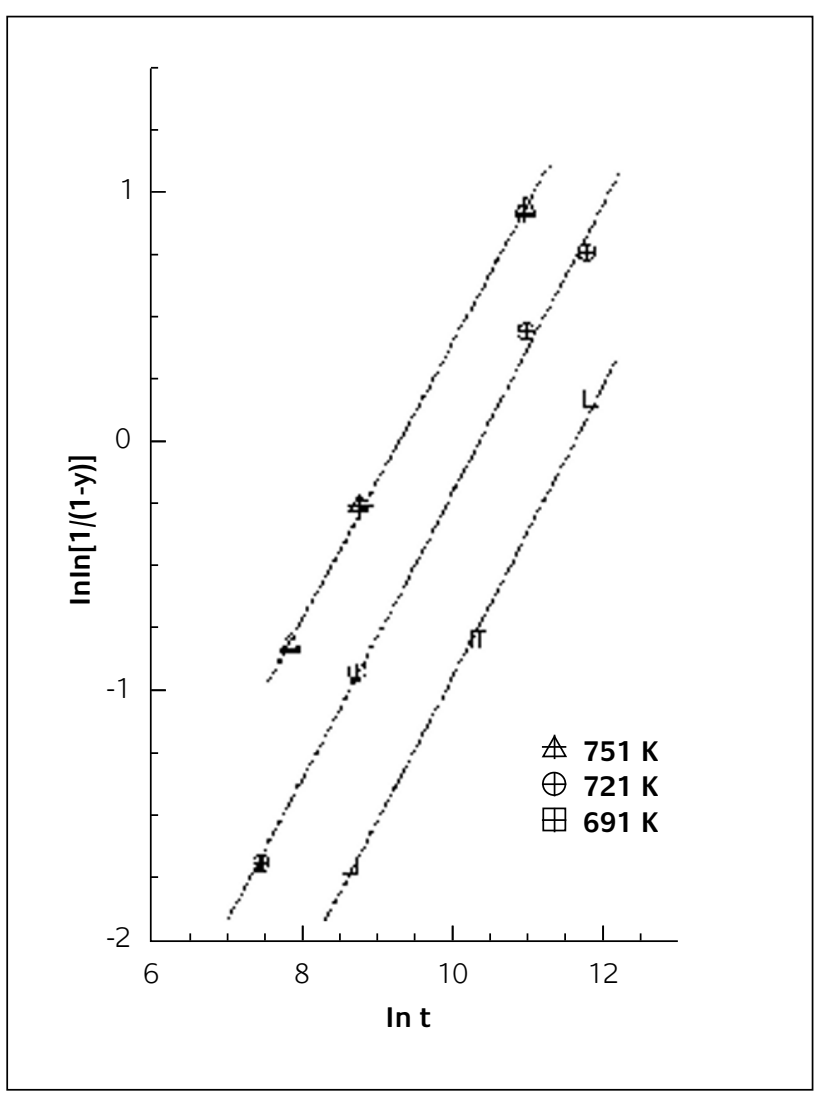

Figure 3

Plot of the Au-7at.\%Pt DMMC transformation of InIn [1/(1-y)] vs. In $t$

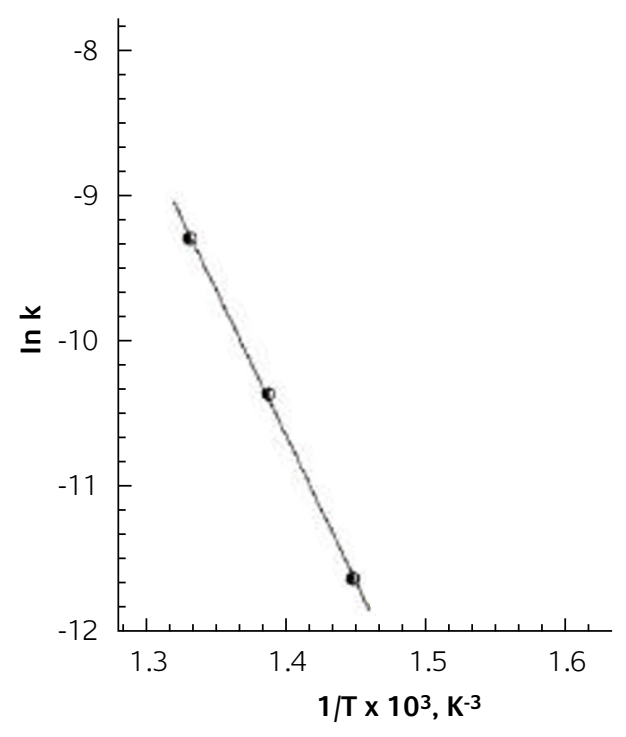

Figure 4

The relation between transformation rates and the reciprocal temperatures of Au-7at.\% Pt DMMC compacted by cold isostatic pressing (CIP) at $172 \mathrm{MPa}$ [11]. The particle sizes of the starting Au and Pt powders were 1.4 to $7.2 \mu \mathrm{m}$ and 1 to $3 \mu \mathrm{m}$, respectively. The CIP compact was sealed for consolidation in an annealed Cu can and then mechanically deformed by extruding, swaging, and wire drawing at room temperature. The heat generated by mechanical processing was removed as quickly as possible after each deformation step by immersing the specimens in cold water to minimize dissolution of the filamentary secondphase Pt into the Au during deformation. The Cu sleeve was removed from the wire with $\mathrm{HNO}_{3}$ acid at a deformation true strain, $\eta$, of 5.8. With the adjustment for collapse of $30 \%$ porosity in the CIP compact, true strain $(\eta)$ was calculated using $\eta=2 \ln \left(d_{o} / d_{f}\right)$, where $d_{o}$ is the initial wire diameter and $d_{f}$ is the final wire diameter. Finally, the sample was wire drawn to $\eta=9.35$ with a mean diameter of $122 \mu \mathrm{m}$. The microstructure and kinetic transformation analyses reported here were all performed on these $122-\mu$ m wires.

Au-Pt wires were sealed in glass ampoules containing an $\mathrm{Ar}$ atmosphere and annealed at various temperatures for various times to achieve isothermal annealing at 691, 721, and 751 $K$. The annealing was performed by placing the ampoules containing the wire specimens in a pre-heated furnace and then removing the ampoules from the furnace after the anneal to air cool. In retrospect it is possible that this annealing procedure may have compromised the accuracy of the activation energies and pre-exponential terms determined, as discussed in the result section. After each anneal, the degree of transformation from the non-equilibrium to the equilibrium solid solution microstructure was determined by electrical resistance measurements performed at $22^{\circ} \mathrm{C}$ by a standard four-probe potentiometric technique [12, 13].

Scanning electron microscope (SEM) specimens were prepared by ordinary metallographic techniques and examined in the unetched condition. A JEOL SEM was used to observe the DMMC microstructure after various annealing times.

Transmission electron microscopy (TEM) was used to resolve the nanofilamentary microstructure of the true strain $=9.35$ wires.

\section{Calculation methods}

The DMMC wires were annealed to study the kinetics of Pt filament dissolution by diffusion into the Au matrix. Annealing transforms the metastable two-phase systems to homogeneous solid solutions, which are the equilibrium states. The transformed fractions of metastable DMMC's to homogeneous solid solution, $y^{\prime}$, are defined as:

$$
y^{\prime}=\frac{\rho_{t}-\rho_{o}}{\rho_{\max }-\rho_{0}}
$$

where $\rho_{0}, \rho_{\mathrm{t}}$, and $\rho_{\max }$ are the resistivitiy before annealing, after annealing for time $t$, and in the homogeneous solid solution condition respectively. 
The empirical homogeneous solid-state transformation considering time and temperature dependence can be expressed by the JMA equation:

$$
y \text { or } y^{\prime}=1-\exp \left[-(k t)^{n}\right]
$$

where $n$ is the power related to the transformation mechanisms, and $k$ is the transformation rate constant of homogenization at a given annealing temperature. The final points on each curve are a value of electrical resistivity very near that of a homogeneous solid solution. It is assumed that the Avrami equation is valid for the transformation of volume fraction from metastable state to homogeneous equilibrium solid solution.

From the JMA equation, the relation of transformed fractions and times can be obtained by taking the double logarithm of Eq. (2) to yield:

$$
\ln \ln \left[\frac{1}{1-y}\right]=n \ln t+n \ln k
$$

The $n$ and $k$ can be obtained by the slope and intercept of the graph. Using an atomistic model to describe the diffusion process, a physical interpretation of the activation energy is determined from the Arrhenius equation;

$$
k=A \exp \left(\frac{-Q}{\mathrm{RT}}\right)
$$

after taking the logarithm of Eq. (4),

$$
\ln k=\frac{-Q}{\mathrm{RT}}+\ln A
$$

where $\mathrm{R}$ is the gas constant, $\mathrm{T}$ is absolute temperature, and $A$ is a constant. The plot of the transformation rate against the reciprocal of annealing temperature yields an empirical activation energy, $Q$, as the slope of the plot. The activation energies are the energies required for diffusive motions in these DMMC systems.

\section{Results}

The isothermal transformation kinetics of Au-7at\%Pt DMMC display the classic sigmoidal shapes shown in Fig. 2. It was assumed that the resistivity changes were directly proportional to the amount of transformation products formed. The kinetic exponent parameters, $n$, can be determined from the straight line plots of $\ln \ln [1 /(1-y)]$ against $\ln t$ shown in Fig. 3. The results show that Au-7at\%Pt yields a mean kinetic exponent of 0.57 in the range of 691-751 $\mathrm{K}$.

The activation energy of Au-7at\%Pt DMMC was calculated from the plot of the relation between transformation rates and temperatures shown in Fig. 4. As Table 1 shows, the activation energy determined by this experiment is lower than the activation energies reported by other investigators for diffusion of Pt in Au and of Au in Pt as measured for single crystal diffusion couples with planar interfaces. It should be noted that the annealing method used may have introduced some error in the determination of $\mathrm{Q}$, since the heating and cooling of the specimens was not instantaneous. The specimens would take a few minutes to reach the furnace temperature when the glass ampoule was first placed in the furnace, and there would be another period when the specimens retained some furnace heat after the ampoule was removed from the furnace and air cooled.

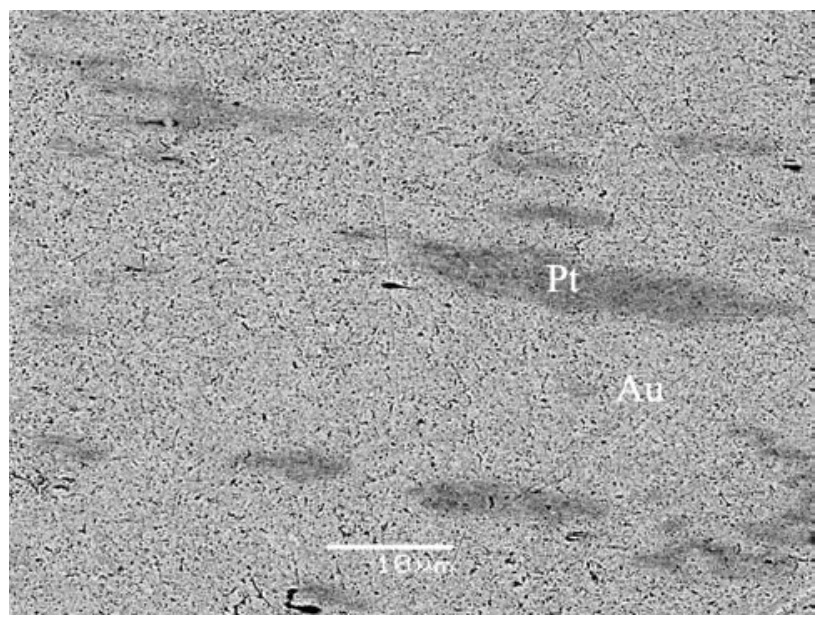

\section{Figure 5}

Back-scattered electron SEM micrograph shows the filamentary microstructure of Pt second phase. The picture was taken in longitudinal cross section. Pt second phases starting to form short filaments in the Au matrix for Au-7at\% Pt DMMC at a wire diameter of $640 \mu \mathrm{m}$

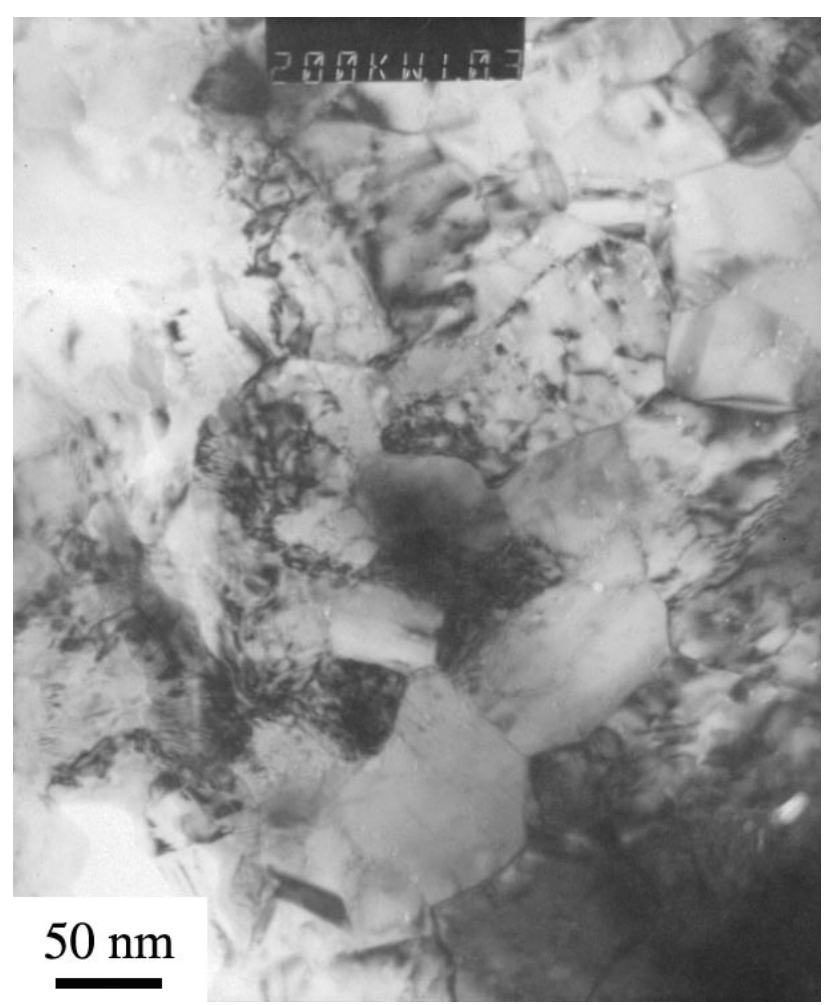

Figure 6

TEM micrograph showing a transverse wire section (i.e., perpendicular to the filaments' long dimension). As wire diameter was reduced by drawing, the Pt filaments became longer and smaller in diameter (30nm in this specimen). 
Fig. 5 shows a SEM backscatter image of the wire draw to $640 \mu \mathrm{m}$ and Fig. 6 shows a TEM image of the wire draw to $122 \mu \mathrm{m}$. As the true strain deformation increases to $\eta=9.3$, the filament diameter decreases to an average value of $30 \mathrm{~nm}$ magnified by TEM images. In all specimens the filaments were observed to develop parallel to the wire axis, in the normal manner for such DMMC's. As annealing progressed, the Pt filaments gradually disappeared by diffusion to form a homogeneous solid solution.

\section{Discussion}

The instability of this composite's microstructure is characterized by the activation energies determined in this study. Factors affecting the activation energy in this complex system include: interface diffusion which has a chemical gradient across the interface; surface diffusion which has a chemical potential from the surface curvature; and a strain effect from coherency energy, stored elastic energy, and high concentrations of defects resulting from plastic deformation. Therefore, the empirical activation energy, Q, can be subdivided into three terms, one for the activation energy of the chemical free energy $Q_{i}$, another for the activation energy of surface curvature, $Q_{c}$, and a third term for the activation energy of the strain effect, $Q_{s}$. The first effect, $Q_{i}$, is clearly seen in the difference between the activation energies in the literature for single crystal planar diffusion of Au-Pt. The latter effect is expected to promote faster diffusion in the system, leading to the observed lower activation energies vis-à-vis published values from single crystal diffusion couple studies. The atomistic approaches for those three parameters are described in the following sections.

\subsection{Chemical free energy}

Table 1 shows that the Au-Pt DMMC activation energy is lower than the activation energy determined by previously published diffusion couple studies. The Au-7at\%Pt DMMC activation energy is 33\% lower than that of the Au-Pt diffusion couple studies. The atomistic interpretation of this disparity is that these differences can be attributed to chemical free energy bonding effects.

\subsection{Surface curvature}

As has been discussed elsewhere [17], the capillary effect may become an important factor in diffusion processes when the particle size becomes small. The Gibbs-Thompson approach can be used to predict the magnitude of the kinetic perturbations involving a curved interface. (The equilibrium condition between two phases in an n-component system is a familiar use.) This approach provides guidance in explaining the effect of filament surface curvature in the transformation from a DMMC microstructure to a homogeneous solid solution. It is assumed that the metastable equilibrium condition between filament and matrix separated by a curved interface can be obtained by equating the chemical potential in the two phases with the curvature effect.

The chemical potential change at the filament-matrix interface can be expressed as:

or,

$$
\mu_{m}\left(T, P_{0}\right)=\mu_{f}\left(T, P_{0}\right)+\gamma \nu \kappa
$$

$$
\mu_{f}\left(T, P_{0}\right)-\mu_{m}\left(T, P_{0}\right)+\gamma \nu_{m} \kappa=0
$$

where $\mu_{f}$ and $\mu_{m}$ are the chemical potential of the second phase filament and of the matrix, respectively, $\gamma$ is interface energy, $\boldsymbol{\kappa}$ is the mean curvature of the interface, $\boldsymbol{v}$ is the molar volume of the filament phase, and $P_{0}$ is the pressure of the filament (for a flat interface, this equals the external pressure on the system.) Eq (6) is valid under the condition $\gamma \kappa \ll P_{0}$.

If it is assumed that the curvature of the DMMC filament is as smooth as a cylinder, then $\kappa=d A / d V$ [17]. In the case of cylindrical $d A / d r=2 \pi h$ and $d V / d r=2 \pi r h$. According to the chain rule $d A / d V=(d A / d r) /(d r / d V)$, we obtain $d A / d V=$ $1 / r$ which allows substitution of interface curvature by $\kappa=$ $1 / r$. The chemical potential will be explained in term of a common thermodynamic property, activity of component $a_{k}$. Therefore, Eq (6) and $\mu_{\kappa}-\mu_{\kappa}^{0} \equiv R T$ In $a_{\kappa}$ can be modified

or

$$
\text { RT In } a_{f}-R T \ln a_{m}=\frac{\gamma V_{m}}{r}
$$

$$
\frac{a_{f}}{a_{m}}=\exp \frac{\gamma V_{m}}{R T r}
$$

and for small values of the exponent

$$
\frac{a_{f}}{a_{m}}=1+\frac{\gamma V_{m}}{\operatorname{RTr}}
$$

Therefore, we can then estimate whether the effect of radius size will be important for the typical filament sizes seen in the Au-Pt DMMC as $\mathrm{T}$ is in Kelvin, $\gamma$ is in $\mathrm{mJ} / \mathrm{m}^{2}$ as well as typical values of $V_{m}=10^{-5} \mathrm{~m}^{3}$ and $R=8.31 \mathrm{~J} \mathrm{~mol}^{-1} \mathrm{~K}^{-1}$, give

Table 1

Comparison of activation values from the literature and activation values determined in this study

\begin{tabular}{lcccc}
\hline System & Q (KJ/mol) & $\begin{array}{c}\text { Temperature } \\
\text { range (K) }\end{array}$ & Reference \\
\hline Au $^{199}$ in Pt diffusion couple & 252 & $853-1265$ & {$[14]$} \\
$\mathrm{Pt}^{195}$ in Au diffusion couple & 254 & $1173-1329$ & {$[15]$} \\
Au-7at.\% Pt DMMC to equilibrium & 167 & $691-751$ & This study \\
\hline
\end{tabular}




$$
\frac{a_{f}}{a_{m}}=1+\frac{1}{r(n m)}
$$

Eq. 9 shows the effect of activity ratio of filaments and matrix. For example, the small filament size range (20-40 $\mathrm{nm}$ ) of this DMMC can significantly increase the ratio of the activity to 1.025-1.05. Thus, to a degree, the size of filament can change the driving force of the transformation of the metastable DMMC to an equilibrium homogeneous solid solution.

\subsection{Strain effect}

There are two major contributions from strain energy to the activation energy of the system. The first is the lattice misfit between the filament and matrix. Lattice misfit between two lattices $(\delta)$ can be approximately defined by:

$$
\delta=\frac{d_{\beta}-d_{\alpha}}{d_{\alpha}}
$$

where $d_{\beta}$ and $d_{\alpha}$ are the unstressed interplanar spacings of matching planes in the filament and matrix phase respectively. Au and Pt are both fcc (CF4) with lattice parameters of 0.4079 $\mathrm{nm}$ and $0.3924 \mathrm{~nm}$ respectively; thus, $\delta=3.0 \%$ for Au-Pt. These small differences suggest that the Au-Pt interface could maintain coherency over moderate distances. The low energy of a coherent interface would be expected to have less effect on changing the activation energy than would a high degree of misfit. Thus, we conclude that the misfit contribution to altering the activation energy is probably small in a Au-Pt DMMC.

Stored energy provides the second contribution from strain energy to the activation energy. High-purity Au quickly undergoes recovery and recrystallization at room temperature after deformation. However, other investigators have reported that heavily deformed Pt (89.5\% reduction) recrystallizes at approximately $698 \mathrm{~K}$ in 15 minutes [18]. Thus, we conclude that the effect of stored energy on altering the activation energy in the Au-Pt DMMC may accelerate diffusive mixing in the DMMC due to the presence of stored energy (i.e. high dislocation density and excess vacancies) in the Pt filaments.

\section{Conclusions}

1. The activation energies for dissolution of nanofilaments of Pt in a Au-7at\%Pt DMMC was found to be $167 \pm 6 \mathrm{~kJ} /$ $\mathrm{mol}$, although the accuracy of these values may have been compromised by the annealing method used, as discussed in the Results section. The empirical activation energy determined in this study for Au-7at\%Pt DMMC is lower than the activation energies determined by other investigators for single crystal, planar interface Au-Pt diffusion couples, presumably due to the effects of the heavy curvature of the Pt filament interfaces with the Au matrix and the many defects present in the DMMC.

2. Transformation of this metastable DMMC to a homogeneous solid solution shows a kinetic exponent, $\mathrm{n}$, of $\sim 0.5$. The physical meaning of diffusivity of DMMC can be explained in terms of the percent of transformation of microstructure with respect to resistivity.

3. We estimate that for filament sizes of $20-40 \mathrm{~nm}$ diameter, the capillary effect would increase the activities of these DMMC's by a factor of 1.025 to 1.05 .

\section{Acknowledgements}

The authors are grateful for the financial support provided by the Royal Thai Government, Thailand; by Kulicke and Soffa, Inc. of Willow Grove, PA, USA; the International Precious Metals Institute of Pensacola, Florida, USA; and by the lowa Space Grant Consortium of the U.S. National Aeronautics and Space Administration. This work was performed at Ames Laboratory, operated for the U.S. Department of Energy by lowa State University under contract no. W-7405-ENG -82. The authors also are grateful to Profs. R. Trivedi, L.S. Chumbley, M.J. Kramer, D. Field, and F. Peters for valuable discussions.

\section{About the authors}

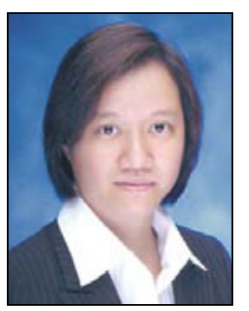

Dr. Wongpreedee is a faculty member at Srinakharinwirot University, Thailand. Her teaching focuses on the physical metallurgy of jewelry alloys. Her research currently involves structure and mechanical property-processing relations of precious metal and alloys.

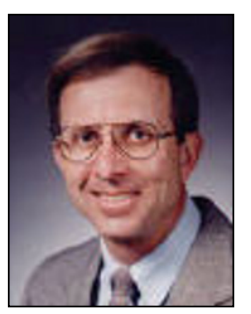

Dr. Russell is Professor of Materials Science and Engineering at lowa State University, USA. His research deals with mechanical properties of intermetallic compounds and deformation processed metal-metal composites.

\section{References}

1 J. D. Verhoeven, F. A. Schmidt, E. D. Gibson and W. A. Spitzig, J.Metals 38 (9) (1986) 20

2 Y. Sakai and H. J. Schneider-Mutau, Acta Mater. 45 (1997) 1017

3 V. Gantovnik, A. M. Russell, L. S. Chumbley, K. Wongpreedee and D. Field, Gold Bull 33(4) (2000) 128

4 A. M. Russell, L. S. Chumbley and Y. Tian, Adv. Engr. Mater. 2 (2000) 11

5 W. A. Spitzig, P. D. Krotz, L. S. Chumbley, H. L. Downing and J. D. Verhoeven, Mater. Res. Soc. Symp. Proc. 120 (1988) 45

6 K.L. Lee, A. F. Whitehouse, A. M. Russell, K. Wongpreedee, S. I. Hong, and P. J. Withers, J. Mater. Sci, 38(16) (2003) 3437 
7 J. C. Malzahn Kampe, T. H. Courtney, Y. Leng, Acta metal. 37(7) (1989) 1735

8 K. Xu, K. Wongpreedee, and A. M. Russell, J. Mater. Sci. 37 (2002) 5209

9 L. Rayleigh, Proc. Lond. Math. Soc. 10(1879) 4

10 W. W. Mullins, Trans. Am. Inst. Min. Metall. Pet. Eng. Inc 218(1960) 354

11 V. B. Gantovnik, M. S.Thesis, lowa State University, 1999

12 S. Hirosawa, T. Sata, J. Yokata and A. Kamio, Mater Trans JIM 19(1) (1998) 139
13 T. Sato, T. Murakami, Y. Amemiva, H. Hashizume and I. Takahashi, Acta Metall 36(5) (1988) 1335

14 G. Rein, H. Mehrer, and K. Maier, Phys. Status Solidii, A45(1978) 253

15 A. J. Mortlock, A. H. Rower, and A. D. Le Claire, Phil. Mag., 5(1960) 803

17 R. K.Trivedi, Lectures on the Theory of Phase Transformations, 2nd edition, H.I. Aaronson, Editor, AIME (2000)

18 E. M. Wise and R. F. Vines, The Platinum Metals and Their Alloys, The International Nickel Company, Inc, NY. (1941) 